\title{
Numerical and Analytical Study for Fourth-Order Integro-Differential Equations Using a Pseudospectral Method
}

\author{
N. H. Sweilam, ${ }^{1}$ M. M. Khader, ${ }^{2}$ and W. Y. Kota ${ }^{3}$ \\ ${ }^{1}$ Department of Mathematics, Faculty of Science, Cairo University, Giza 12613, Egypt \\ ${ }^{2}$ Department of Mathematics, Faculty of Science, Benha University, Benha 13511, Egypt \\ ${ }^{3}$ Department of Mathematics, Faculty of Science, Mansoura University, Damietta 35516, Egypt
}

Correspondence should be addressed to M. M. Khader; mohamedmbd@yahoo.com

Received 16 July 2012; Accepted 2 December 2012

Academic Editor: Pedro Ribeiro

Copyright (c) 2013 N. H. Sweilam et al. This is an open access article distributed under the Creative Commons Attribution License, which permits unrestricted use, distribution, and reproduction in any medium, provided the original work is properly cited.

\begin{abstract}
A numerical method for solving fourth-order integro-differential equations is presented. This method is based on replacement of the unknown function by a truncated series of well-known shifted Chebyshev expansion of functions. An approximate formula of the integer derivative is introduced. The introduced method converts the proposed equation by means of collocation points to system of algebraic equations with shifted Chebyshev coefficients. Thus, by solving this system of equations, the shifted Chebyshev coefficients are obtained. Special attention is given to study the convergence analysis and derive an upper bound of the error of the presented approximate formula. Numerical results are performed in order to illustrate the usefulness and show the efficiency and the accuracy of the present work.
\end{abstract}

\section{Introduction}

The integro-differential equation (IDE) is an equation that involves both integrals and derivatives of an unknown function. Mathematical modeling of real-life problems usually results in functional equations, like ordinary or partial differential equations, and integral and integro-differential equations, stochastic equations. Many mathematical formulations of physical phenomena contain integro-differential equations; these equations arise in many fields like physics, astronomy, potential theory, fluid dynamics, biological models, and chemical kinetics. Integro-differential equations; are usually difficult to solve analytically; so, it is required to obtain an efficient approximate solution [1-5]. Recently, several numerical methods to solve IDEs have been given such as variational iteration method $[6,7]$, homotopy perturbation method $[8,9]$, spline functions expansion $[10,11]$, and collocation method [12-15].

Chebyshev polynomials are well-known family of orthogonal polynomials on the interval $[-1,1]$ that have many applications $[4,6,8,13]$. They are widely used because of their good properties in the approximation of functions. However, with our best knowledge, very little work was done to adapt these polynomials to the solution of integro-differential equations. Orthogonal polynomials have a great variety and wealth of properties. Some of these properties take a very concise form in the case of the Chebyshev polynomials, making Chebyshev polynomials of leading importance among orthogonal polynomials. The Chebyshev polynomials belong to an exclusive band of orthogonal polynomials, known as Jacobi polynomials, which correspond to weight functions of the form $(1-x)^{\alpha}(1+x)^{\beta}$ and which are solutions of SturmLiouville equations [16].

In this work, we derive an approximate formula of the integral derivative $y^{(n)}(x)$ and derive an upper bound of the error of this formula, and then we use this formula to solve a class of two-point boundary value problems (BVPs) for the fourth-order integro-differential equations as

$$
\begin{array}{r}
y^{(i v)}(x)=f(x)+\gamma y(x)+\int_{0}^{x}[p(t) y(t)+q(t) \Theta(y(t))] d t, \\
0 \leq x, t \leq 1,
\end{array}
$$


under the boundary and initial conditions

$$
\begin{array}{ll}
y(0)=\alpha_{0}, & y^{\prime \prime}(0)=\alpha_{1}, \\
y(1)=\beta_{0}, & y^{\prime \prime}(1)=\beta_{1},
\end{array}
$$

where $f(x), p(x)$, and $q(x)$ are known functions and $\gamma, \alpha_{0}$, $\alpha_{1}, \beta_{0}$, and $\beta_{1}$ are suitable constants. Several numerical methods to solve the fourth-order integro-differential equations have been given such as Chebyshev cardinal functions [17], variational iteration method [7], and others.

\section{Some Basic Properties and Derivation of an Approximate Formula of the Derivative for Chebyshev Polynomials Expansion}

The Chebyshev polynomial of the first kind is a polynomial in $z$ of degree $n$, defined by the relation

$$
T_{n}(z)=\cos n \theta, \quad \text { when } z=\cos \theta .
$$

The Chebyshev polynomials of degree $n>0$ of the first kind have precisely $n$ zeros and $n+1$ local extrema in the interval $[-1,1]$. The zeros of $T_{n}(z)$ are denoted by

$$
z_{k}=\cos \frac{(k-1 / 2) \pi}{n}, \quad k=1,2, \ldots, n .
$$

The Chebyshev polynomials can be determined with the aid of the following recurrence formula [18]:

$$
\begin{gathered}
T_{n+1}(z)=2 z T_{n}(z)-T_{n-1}(z), \\
T_{0}(z)=1, \quad T_{1}(z)=z, \quad n=1,2, \ldots
\end{gathered}
$$

The analytic form of the Chebyshev polynomials $T_{n}(z)$ of degree $n$ is given by

$$
T_{n}(z)=n \sum_{i=0}^{[n / 2]}(-1)^{i} 2^{n-2 i-1} \frac{(n-i-1) !}{(i) !(n-2 i) !} z^{n-2 i},
$$

where $[n / 2]$ denotes the integer part of $n / 2$. The orthogonality condition is

$$
\int_{-1}^{1} \frac{T_{i}(z) T_{j}(z)}{\sqrt{1-z^{2}}} d x= \begin{cases}\pi, & \text { for } i=j=0 \\ \frac{\pi}{2}, & \text { for } i=j \neq 0 \\ 0, & \text { for } i \neq j .\end{cases}
$$

In order to use these polynomials on the interval $[0,1]$, we define the so called shifted Chebyshev polynomials by introducing the change of variable $z=2 x-1$. The shifted Chebyshev polynomials are denoted by $T_{n}^{*}(x)$ and defined as $T_{n}^{*}(x)=T_{n}(2 x-1)=T_{2 n}(\sqrt{x})$.

The function $y(x)$, which belongs to the space of square integrable in $[0,1]$, may be expressed in terms of shifted Chebyshev polynomials as

$$
y(x)=\sum_{i=0}^{\infty} c_{i} T_{i}^{*}(x),
$$

where the coefficients $c_{i}$ are given by

$$
\begin{array}{r}
c_{0}=\frac{1}{\pi} \int_{0}^{1} \frac{y(x) T_{0}^{*}(x)}{\sqrt{x-x^{2}}} d x, \quad c_{i}=\frac{2}{\pi} \int_{0}^{1} \frac{y(x) T_{i}^{*}(x)}{\sqrt{x-x^{2}}} d x, \\
i=1,2, \ldots
\end{array}
$$

In practice, only the first $(m+1)$ terms of shifted Chebyshev polynomials are considered. Then, we have that

$$
y_{m}(x)=\sum_{i=0}^{m} c_{i} T_{i}^{*}(x) .
$$

Lemma 1. The analytic form of the shifted Chebyshev polynomials $T_{n}^{*}(x)$ of degree $n$ is given by

$$
T_{n}^{*}(x)=n \sum_{k=0}^{n}(-1)^{n-k} \frac{2^{2 k}(n+k-1) !}{(2 k) !(n-k) !} x^{k}, \quad n=1,2, \ldots
$$

Proof. Since we have $T_{n}^{*}(x)=T_{2 n}(\sqrt{x})$, then by substituting in (6), we can obtain that

$$
\begin{array}{r}
T_{n}^{*}(x)=2 n \sum_{i=0}^{n}(-1)^{i} \frac{2^{2 n-2 i-1}(2 n-i-1) !}{(i) !(2 n-2 i) !} x^{n-i}, \\
n=1,2, \ldots
\end{array}
$$

Now, we put $k=n-i$ in (12) we obtain the desired result (11).

The main approximate formula of the derivative of $y_{m}(x)$, and is given in the following theorem.

Theorem 2. Let $y(x)$ be approximated by shifted Chebyshev polynomials as (10), and also suppose that $r$ is integer; then,

$$
D^{r}\left(y_{m}(x)\right)=\sum_{i=r}^{m} \sum_{k=r}^{i} c_{i} \lambda_{i, k, r} x^{k-r},
$$

where $\lambda_{i, k, r}$ is given by

$$
\lambda_{i, k, r}=(-1)^{i-k} \frac{2^{2 k} i(i+k-1) ! k !}{(i-k) !(2 k) !(k-r) !} .
$$

Proof. Since the differential operator $D^{r}$ is linear, we can obtain that

$$
D^{r}\left(y_{m}(x)\right)=\sum_{i=0}^{m} c_{i} D^{r}\left(T_{i}^{*}(x)\right) .
$$

Since $D^{r} c=0, c$ is a constant, and

$$
D^{r} x^{n}= \begin{cases}0, & \text { for } n \in N, n<r, \\ \frac{n !}{(n-r) !} x^{n-r}, & \text { for } n \in N, n \geq r .\end{cases}
$$

Then, we have that

$$
D^{r} T_{i}^{*}(x)=0, \quad i=0,1, \ldots, r-1,
$$


and for $i=r, r+1, \ldots, m$, and by using (16), we get that

$$
\begin{aligned}
D^{r} T_{i}^{*}(x) & =i \sum_{k=r}^{i}(-1)^{i-k} \frac{2^{2 k}(i+k-1) !}{(i-k) !(2 k) !} D^{r} x^{k} \\
& =i \sum_{k=r}^{i}(-1)^{i-k} \frac{2^{2 k}(i+k-1) ! k !}{(i-k) !(2 k) !(k-r) !} x^{k-r} .
\end{aligned}
$$

A combination of (17), (18), and (14) leads to the desired result and completes the proof of the theorem.

\section{Error Analysis}

In this section, special attention is given to study the convergence analysis and evaluate the upper bound of the error of the proposed formula.

Theorem 3 (Chebyshev truncation theorem; see [18]). The error in approximating $y(x)$ by the sum of its first $m$ terms is bounded by the sum of the absolute values of all the neglected coefficients. If

$$
y_{m}(x)=\sum_{k=0}^{m} c_{k} T_{k}(x)
$$

then

$$
E_{T}(m) \equiv\left|y(x)-y_{m}(x)\right| \leq \sum_{k=m+1}^{\infty}\left|c_{k}\right|
$$

for all $y(x)$, all $m$, and all $x \in[-1,1]$.

Theorem 4. The derivative of order $r$ for the shifted Chebyshev polynomials can be expressed in terms of the shifted Chebyshev polynomials themselves in the following form:

$$
D^{r}\left(T_{i}^{*}(x)\right)=\sum_{k=r}^{i} \sum_{j=0}^{k-r} \Theta_{i, j, k} T_{j}^{*}(x),
$$

where

$$
\begin{array}{r}
\Theta_{i, j, k}=\frac{(-1)^{i-k} 2 i(i+k-1) ! \Gamma(k-r+1 / 2)}{h_{j} \Gamma(k+1 / 2)(i-k) !(k-r-j) !(k+j-r) !}, \\
h_{0}=2, h_{j}=1, j=0,1, \ldots
\end{array}
$$

Proof. We use the properties of the shifted Chebyshev polynomials [18] and expand $x^{k-r}$ in (18) in the following form:

$$
x^{k-r}=\sum_{j=0}^{k-r} c_{k j} T_{j}^{*}(x),
$$

where $c_{k j}$ can be obtained using (9), and $y(x)=x^{k-r}$; then,

$$
\begin{array}{r}
c_{k j}=\frac{2}{h_{j} \pi} \int_{0}^{1} \frac{x^{k-r} T_{j}^{*}(x)}{\sqrt{x-x^{2}}} d x, \quad h_{0}=2, h_{j}=1, \\
j=1,2, \ldots
\end{array}
$$

At $j=0$, we find that $c_{k 0}=(1 / \pi) \int_{0}^{1}\left(x^{k-r} T_{0}^{*}(x) /\right.$ $\left.\sqrt{x-x^{2}}\right) d x=(1 / \sqrt{\pi})(\Gamma(k-r+1 / 2) /(k-r) !)$; also, at any $j$ and using the formula (10), we can find that

$$
\begin{array}{r}
c_{k j}=\frac{j}{\sqrt{\pi}} \sum_{l=0}^{j}(-1)^{j-l} \frac{(j+l-1) ! 2^{2 l+1} \Gamma(k+l-r+1 / 2)}{(j-l) !(2 l) !(k+l-r) !}, \\
j=1,2,3, \ldots,
\end{array}
$$

employing (18) and (23) gives

$$
D^{r}\left(T_{i}^{*}(x)\right)=\sum_{k=r}^{i} \sum_{j=0}^{k-r} \Theta_{i, j, k} T_{j}^{*}(x), \quad i=r, r+1, \ldots,
$$

where

$$
\Theta_{i, j, k}= \begin{cases}i \frac{(-1)^{i-k}(i+k-1) ! 2^{2 k} k ! \Gamma(k-r+1 / 2)}{(i-k) !(2 k) ! \sqrt{\pi}(\Gamma(k+1-r))^{2}}, & j=0 ; \\ \frac{(-1)^{i-k} i j(i+k-1) ! 2^{2 k+1} k !}{\sqrt{\pi}(k-r) !(i-k) !(2 k) !} & \\ \quad \times \sum_{l=0}^{j} \frac{(-1)^{j-l}(j+l-1) ! 2^{2 l} \Gamma(k+l-r+1 / 2)}{(j-l) !(2 l) !(k+l-r) !}, & j=1,2,3, \ldots\end{cases}
$$

After some lengthy manipulation, $\Theta_{i, j, k}$ can be put in the following form:

$$
\begin{array}{r}
\Theta_{i, j, k}=\frac{(-1)^{i-k} 2 i(i+k-1) ! \Gamma(k-r+1 / 2)}{h_{j} \Gamma(k+1 / 2)(i-k) !(k-r-j) !(k+j-r) !}, \\
j=0,1, \ldots,
\end{array}
$$

and this completes the proof of the theorem.

Theorem 5. The error $\left|E_{T}(m)\right|=\left|D^{r} y(x)-D^{r} y_{m}(x)\right|$ in approximating $D^{r} y(x)$ by $D^{r} y_{m}(x)$ is bounded by

$$
\left|E_{T}(m)\right| \leq\left|\sum_{i=m+1}^{\infty} c_{i}\left(\sum_{k=r}^{i} \sum_{j=0}^{k-r} \Theta_{i, j, k}\right)\right|
$$

Proof. A combination of (8), (10), and (21) leads to

$$
\begin{aligned}
\left|E_{T}(m)\right| & =\left|D^{r} y(x)-D^{r} y_{m}(x)\right| \\
& =\left|\sum_{i=m+1}^{\infty} c_{i}\left(\sum_{k=r}^{i} \sum_{j=0}^{k-r} \Theta_{i, j, k} T_{j}^{*}(x)\right)\right|,
\end{aligned}
$$

but $\left|T_{j}^{*}(x)\right| \leq 1$; so, we can obtain that

$$
\left|E_{T}(m)\right| \leq\left|\sum_{i=m+1}^{\infty} c_{i}\left(\sum_{k=r}^{i} \sum_{j=0}^{k-r} \Theta_{i, j, k}\right)\right|
$$

and subtracting the truncated series from the infinite series, bounding each term in the difference, and summing the bounds complete the proof of the theorem. 


\section{Procedure Solution for the Fourth-Order Integro-Differential Equation}

In this section, we will present the proposed method to solve numerically the fourth-order integro-differential equation of the form in (1). The unknown function $y(x)$ may be expanded by finite series of shifted Chebyshev polynomials as in the following approximation:

$$
y_{m}(x)=\sum_{n=0}^{m} c_{n} T_{n}^{*}(x)
$$

and approximated formula of its derivatives can be defined in Theorem 2. From (1), (32), and Theorem 2, we have that

$$
\begin{aligned}
& \sum_{i=r}^{m} \sum_{k=r}^{i} c_{i} \lambda_{i, k, r} x^{k-r} \\
&=f(x)+\gamma \sum_{n=0}^{m} c_{n} T_{n}^{*}(x) \\
&+\int_{0}^{x}\left[p(t)\left(\sum_{n=0}^{m} c_{n} T_{n}^{*}(t)\right)\right. \\
&\left.\quad+q(t) \Theta\left(\sum_{n=0}^{m} c_{n} T_{n}^{*}(t)\right)\right] d t .
\end{aligned}
$$

We now collocate $(33)$ at $(m-1+r)$ points $x_{s}, s=$ $0,1, \ldots, m-r$ as

$$
\begin{aligned}
& \sum_{i=4}^{m} \sum_{k=4}^{i} c_{i} \lambda_{i, k, 4} x_{s}^{k-4} \\
&=f\left(x_{s}\right)+\gamma \sum_{n=0}^{m} c_{n} T_{n}^{*}\left(x_{s}\right) \\
&+\int_{0}^{x_{s}}\left[p(t)\left(\sum_{n=0}^{m} c_{n} T_{n}^{*}(t)\right)\right. \\
&\left.+q(t) \Theta\left(\sum_{n=0}^{m} c_{n} T_{n}^{*}(t)\right)\right] d t .
\end{aligned}
$$

For suitable collocation points, we use roots of shifted Chebyshev polynomial $T_{m+1-r}^{*}(x)$. The integral terms in (34) can be found using composite trapezoidal integration technique as

$$
\begin{gathered}
\int_{0}^{x_{s}}\left[p(t)\left(\sum_{n=0}^{m} c_{n} T_{n}^{*}(t)\right)+q(t) \Theta\left(\sum_{n=0}^{m} c_{n} T_{n}^{*}(t)\right)\right] d t \\
\cong \frac{h_{s}}{2}\left(\Omega\left(t_{0}\right)+\Omega\left(t_{L}\right)+2 \sum_{k=1}^{L-1} \Omega\left(t_{k}\right)\right),
\end{gathered}
$$

where $\Omega(t)=p(t) \sum_{n=0}^{m} c_{n} T_{n}^{*}(t)+q(t) \Theta\left(\sum_{n=0}^{m} c_{n} T_{n}^{*}(t)\right), h_{s}=$ $x_{s} / L$, for an arbitrary integer $L, t_{j+1}=t_{j}+h_{s}, s=0,1, \ldots$, $m-r$, and $j=0,1, \ldots, L$. So, by using (34) and (35), we obtain

$$
\begin{aligned}
\sum_{i=4}^{m} \sum_{k=4}^{i} c_{i} \lambda_{i, k, r} x_{s}^{k-r} & \\
= & f\left(x_{s}\right)+\gamma \sum_{n=0}^{m} c_{n} T_{n}^{*}\left(x_{s}\right) \\
& +\frac{h_{s}}{2}\left(\Omega\left(t_{0}\right)+\Omega\left(t_{L}\right)+2 \sum_{k=1}^{L-1} \Omega\left(t_{k}\right)\right) .
\end{aligned}
$$

Also, by substituting (32) in the boundary conditions (2), we can obtain $r$ equations as follows:

$$
\begin{gathered}
\sum_{i=0}^{m}(-1)^{i} c_{i}=\alpha_{0}, \quad \sum_{i=0}^{m} c_{i}=\beta_{0}, \\
\sum_{i=2}^{m} c_{i} T_{i}^{* \prime \prime}(0)=\alpha_{1}, \quad \sum_{i=2}^{m} c_{i} T_{i}^{* \prime \prime}(1)=\beta_{1} .
\end{gathered}
$$

Equation (36), together with $r$ equations of the boundary conditions (37), give $(m+1)$ of system of algebraic equations which can be solved, for the unknowns $c_{n}, n=$ $0,1, \ldots, m$, using conjugate gradient method or Newton iteration method.

\section{Numerical Results}

In this section, to verify the validity and the accuracy and support our theoretical discussion of the proposed method, we give some computations results of numerical examples.

Example 6. Consider the nonlinear fourth-order integrodifferential equation as in (1) and (2) with $f(x)=1, \gamma=$ $0, p(t)=0, q(t)=e^{-t}$, and $\Theta(y)=y^{2}(x)$; then, the integro-differential equation will be

$$
y^{(i v)}(x)=1+\int_{0}^{x} e^{-t} y^{2}(t) d t, \quad 0 \leq x \leq 1,
$$

subject to the boundary conditions

$$
y(0)=y^{\prime \prime}(0)=1, \quad y(1)=y^{\prime \prime}(1)=e .
$$

The exact solution of this problem is $y(x)=e^{x}$ [7].

We apply the suggested method with $m=5$ and approximate the solution $y(x)$ as follows:

$$
y_{5}(x)=\sum_{n=0}^{5} c_{n} T_{n}^{*}(x) \text {. }
$$

From (38), (40), and Theorem 2, we have that

$$
\sum_{i=4}^{5} \sum_{k=4}^{i} c_{i} \lambda_{i, k, 4} x^{k-4}=1+\int_{0}^{x} e^{-t}\left(\sum_{n=0}^{5} c_{n} T_{n}^{*}(t)\right)^{2} d t .
$$

We now collocate (41) at points, $x_{s}, s=0,1$ as

$$
\sum_{i=4}^{5} \sum_{k=4}^{i} c_{i} \lambda_{i, k, 4} x_{s}^{k-4}=1+\int_{0}^{x_{s}} e^{-t}\left(\sum_{n=0}^{5} c_{n} T_{n}^{*}(t)\right)^{2} d t .
$$


For suitable collocation points we use roots of shifted Chebyshev polynomial $T_{2}^{*}(x)$. The integral terms in (42) can be found using composite trapezoidal integration technique as

$$
\begin{aligned}
\int_{0}^{x_{s}} e^{-t} & \left(\sum_{n=0}^{5} c_{n} T_{n}^{*}(t)\right)^{2} d t \\
& =\frac{h_{s}}{2}\left(\Omega\left(t_{0}\right)+\Omega\left(t_{L}\right)+2 \sum_{k=1}^{L-1} \Omega\left(t_{k}\right)\right),
\end{aligned}
$$

where $\Omega(t)=e^{-t}\left(\sum_{n=0}^{5} c_{n} T_{n}^{*}(t)\right)^{2}, h_{s}=x_{s} / L$, for an arbitrary integer $L, t_{j+1}=t_{j}+h_{s}, s=0,1$, and $j=0,1, \ldots, L$. So, by using (43) and (42), we obtain

$$
\begin{aligned}
& \sum_{i=4}^{5} \sum_{k=4}^{i} c_{i} \lambda_{i, k, 4} x_{s}^{k-4} \\
& \quad=1+\frac{h_{s}}{2}\left(\Omega\left(t_{0}\right)+\Omega\left(t_{L}\right)+2 \sum_{k=1}^{L-1} \Omega\left(t_{k}\right)\right) .
\end{aligned}
$$

Also, by substituting (40) in the boundary conditions (39), we can obtain four equations as follows:

$$
\begin{gathered}
c_{0}-c_{1}+c_{2}-c_{3}+c_{4}-c_{5}=1, \\
c_{0}+c_{1}+c_{2}+c_{3}+c_{4}+c_{5}=e, \\
l_{0} c_{0}+l_{1} c_{1}+l_{2} c_{2}+l_{3} c_{3}+l_{4} c_{4}+l_{5} c_{5}=1, \\
s_{0} c_{0}+s_{1} c_{1}+s_{2} c_{2}+s_{3} c_{3}+s_{4} c_{4}+s_{5} c_{5}=e,
\end{gathered}
$$

where $l_{i}=T_{i}^{* \prime \prime}(0)$ and $s_{i}=T_{i}^{* \prime \prime}(1)$.

Equation (44), together with four equations of the boundary conditions (45), represent, a nonlinear system of six algebraic equations in the coefficients $c_{n}$; by solving it using the Newton iteration method, we obtain

$$
\begin{array}{lll}
c_{0}=1.75379, & c_{1}=0.85039, & c_{2}=0.10478, \\
c_{3}=0.00872, & c_{4}=0.00057, & c_{5}=0.00003 .
\end{array}
$$

The behavior of the approximate solution using the proposed method with $m=5$, the approximate solution using variational iteration method (VIM), and the exact solution are presented in Figure 1. Table 1 shows the behavior of the absolute error between exact solution and approximate solution using the presented method at $m=6$ and $m=8$. From Figure 1 and Table 1, it is clear that the proposed method can be considered as an efficient method to solve the nonlinear integro-differential equations. Table 1 indicates that as $m$ increases the errors decrease more rapidly; hence, for better results, using number $m$ is recommended. Also, we can conclude that the obtained approximated solution is in excellent agreement with the exact solution.

Example 7. Consider the linear fourth-order integrodifferential equation as in (1) and (2) with $f(x)=x+(x+3) e^{x}$,

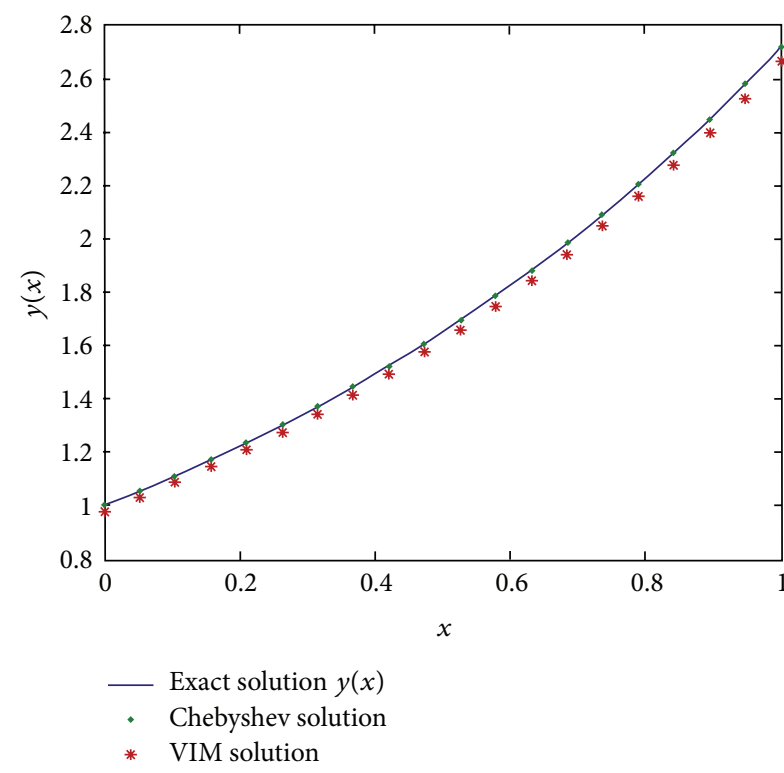

FIgURE 1: The behavior of the exact solution, the approximate solution using VIM, and the approximate solution using the proposed method at $m=5$.

$\gamma=1, p(t)=-1, h(t)=0$, and $\Theta(y)=y(x)$; then, the integro-differential equation will be

$$
\begin{aligned}
y^{(i v)}(x)=x & +(x+3) e^{x}+y(x) \\
- & \int_{0}^{x} y(t) d t, \quad 0 \leq x \leq 1
\end{aligned}
$$

subject to the boundary conditions

$$
\begin{array}{cl}
y(0)=1, & (1)=1+e, \\
y^{\prime \prime}(0)=2, & y^{\prime \prime}(1)=3 e .
\end{array}
$$

The exact solution of this problem is $y(x)=1+x e^{x}$ [17].

We apply the suggested method with $m=5$ and approximate the solution $y(x)$ as follows:

$$
y(x) \cong \sum_{n=0}^{5} c_{n} T_{n}^{*}(x) .
$$

By the same procedure in the previous example, we have

$$
\begin{aligned}
\sum_{i=4}^{5} \sum_{k=4}^{i} c_{i} \lambda_{i, k, 4} x_{s}^{k-4} & \\
= & f\left(x_{s}\right)+\sum_{n=0}^{5} c_{n} T_{n}^{*}\left(x_{s}\right)+\frac{h_{s}}{2} \\
& \times\left(\Omega\left(t_{0}\right)+\Omega\left(t_{L}\right)+2 \sum_{k=1}^{L-1} \Omega\left(t_{k}\right)\right), \quad s=0,1,2,
\end{aligned}
$$


TABLE 1: The behavior of the absolute error between the exact solution and approximate solution at $m=6$ and $m=8$.

\begin{tabular}{lcc}
\hline$x$ & $\left|y_{\text {ex. }}-y_{\text {ap. }}\right|$ at $m=6$ & $\left|y_{\text {ex. }}-y_{\text {ap. }}\right|$ at $m=8$ \\
\hline 0.0 & $2.2548 e-10$ & $2.0254 e-10$ \\
0.2 & $2.3654 e-04$ & $1.2548 e-06$ \\
0.4 & $3.5687 e-04$ & $3.2541 e-06$ \\
0.6 & $0.1587 e-04$ & $5.2548 e-06$ \\
0.8 & $9.2450 e-04$ & $7.2581 e-06$ \\
1.0 & $1.2589 e-10$ & $2.2548 e-10$ \\
\hline
\end{tabular}

TABLE 2: The behaviour of the absolute error between the exact solution and approximate solution at $m=7$ and $m=9$.

\begin{tabular}{lcc}
\hline$x$ & $\left|y_{\text {ex. }}-y_{\text {ap. }}\right|$ at $m=7$ & $\left|y_{\text {ex. }}-y_{\text {ap. }}\right|$ at $m=9$ \\
\hline 0.0 & $1.2587 e-08$ & $5.1236 e-09$ \\
0.2 & $6.2548 e-03$ & $2.2258 e-05$ \\
0.4 & $2.0254 e-03$ & $9.2154 e-05$ \\
0.6 & $1.3654 e-03$ & $2.0054 e-05$ \\
0.8 & $0.2540 e-03$ & $2.3690 e-05$ \\
1.0 & $6.0254 e-08$ & $5.2478 e-09$ \\
\hline
\end{tabular}

where $\Omega(t)=\sum_{n=0}^{5} c_{n} T_{n}^{*}(t)$, and the nodes $t_{j+1}=t_{j}+h_{s}, j=$ $0,1, \ldots, L, t_{0}=0$, and $h_{s}=x_{s} / L$. We can write the initialboundary conditions in the form

$$
\begin{gathered}
c_{0}-c_{1}+c_{2}-c_{3}+c_{4}-c_{5}+c_{6}=1, \\
c_{0}+c_{1}+c_{2}+c_{3}+c_{4}+c_{5}+c_{6}=1+e, \\
l_{0} c_{0}+l_{1} c_{1}+l_{2} c_{2}+l_{3} c_{3}+l_{4} c_{4}+l_{5} c_{5}+l_{6} c_{6}=2, \\
s_{0} c_{0}+s_{1} c_{1}+s_{2} c_{2}+s_{3} c_{3}+s_{4} c_{4}+s_{5} c_{5}+s_{6} c_{6}=3 e .
\end{gathered}
$$

By using (50) and (51), we obtain a linear system of seven algebraic equations in the coefficients $c_{n}$; by solving it using the conjugate gradient method, we obtain

$$
\begin{array}{rlrl}
c_{0} & =2.09189, & c_{1}=1.32820, & c_{2}=0.26461, \\
c_{3}=0.03079, & c_{4}=0.00264, & c_{5}=0.00015 .
\end{array}
$$

The behavior of the approximate solution using the proposed method with $m=6$, the approximate solution using variational iteration method (VIM) and the exact solution are presented in Figure 2. Table 2 shows the behaviour of the absolute error between exact solution and approximate solution using the presented method at $m=7$ and $m=$ 9. From this figure, it is clear that the proposed method can be considered as an efficient method to solve the linear integro-differential equations. Also, we can conclude that the obtained approximate solution is in excellent agreement with the exact solution.

\section{Conclusion and Discussion}

Integro-differential equations are usually difficult to solve analytically; so, it is required to obtain the approximate solution. In this paper, we proposed the pseudospectral method

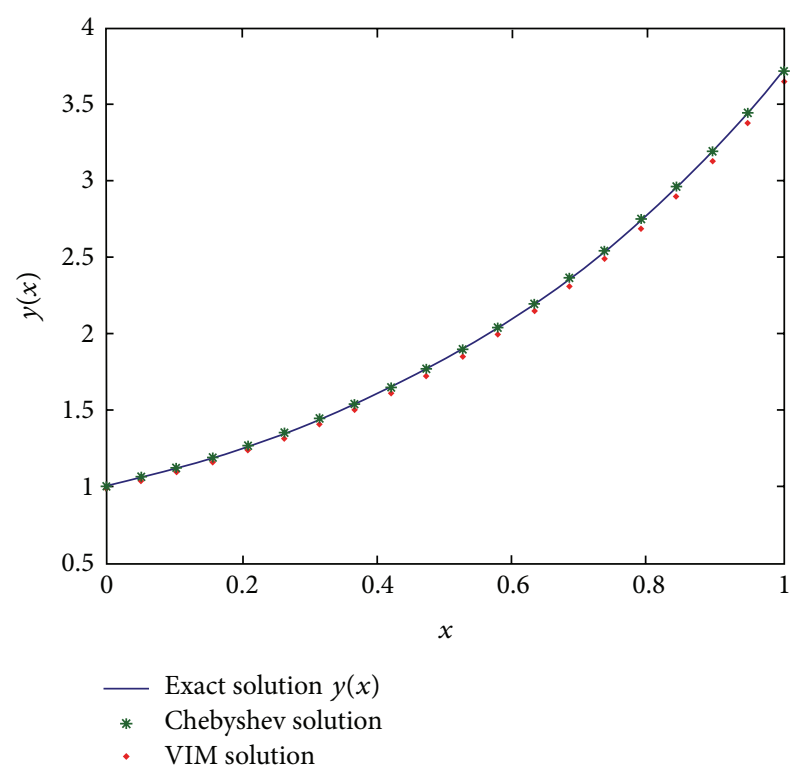

FIgURE 2: The behavior of the exact solution, the approximate solution using VIM, and the approximate solution using the proposed method at $m=6$.

using shifted Chebyshev method for solving the integrodifferential equations. The Chebyshev method is useful for acquiring both the general solution and particular solution as demonstrated in examples. Special attention is given to study the convergence analysis and derive an upper bound of the error of the derived approximate formula. From our obtained results, we can conclude that the proposed method gives solutions in excellent agreement with the exact solution and better than the other methods. An interesting feature of this method is that when an integral system has linearly independent polynomial solution of degree $m$ or less than $m$, the method can be used for finding the analytical solution. All computations are done using MATLAB 8.

\section{References}

[1] R. P. Agarwal, "Boundary value problems for higher order integro-differential equations," Nonlinear Analysis: Theory, Methods \& Applications, vol. 7, no. 3, pp. 259-270, 1983.

[2] E. Babolian, F. Fattahzadeh, and E. G. Raboky, "A Chebyshev approximation for solving nonlinear integral equations of Hammerstein type," Applied Mathematics and Computation, vol. 189, no. 1, pp. 641-646, 2007.

[3] A. H. Borzabadi, A. V. Kamyad, and H. H. Mehne, "A different approach for solving the nonlinear Fredholm integral equations of the second kind," Applied Mathematics and Computation, vol. 173, no. 2, pp. 724-735, 2006.

[4] M. M. Khader, "On the numerical solutions for the fractional diffusion equation," Communications in Nonlinear Science and Numerical Simulation, vol. 16, no. 6, pp. 2535-2542, 2011.

[5] N. H. Sweilam, M. M. Khader, and A. M. Nagy, "Numerical solution of two-sided space-fractional wave equation using finite difference method," Journal of Computational and Applied Mathematics, vol. 235, no. 8, pp. 2832-2841, 2011. 
[6] M. M. Khader, "Introducing an efficient modification of the variational iteration method by using Chebyshev polynomials," Applications and Applied Mathematics, vol. 7, no. 1, pp. 283-299, 2012.

[7] N. H. Sweilam, "Fourth order integro-differential equations using variational iteration method," Computers \& Mathematics with Applications, vol. 54, no. 7-8, pp. 1086-1091, 2007.

[8] M. M. Khader, "Introducing an efficient modification of the homotopy perturbation method by using Chebyshev polynomials," Arab Journal of Mathematical Sciences, vol. 18, no. 1, pp. 61-71, 2012.

[9] N. H. Sweilam, M. M. Khader, and R. F. Al-Bar, "Homotopy perturbation method for linear and nonlinear system of fractional integro-differential equations," International Journal of Computational Mathematics and Numerical Simulation, vol. 1, no. 1, pp. 73-87, 2008.

[10] M. M. Khader and S. T. Mohamed, "Numerical treatment for first order neutral delay differential equations using spline functions," Engineering Mathematics Letters, vol. 1, no. 1, pp. 3243, 2012.

[11] S. T. Mohamed and M. M. Khader, "Numerical solutions to the second order Fredholm integro-differential equations using the spline functions expansion," Global Journal of Pure and Applied Mathematics, vol. 34, pp. 21-29, 2011.

[12] M. M. Khader and A. S. Hendy, "The approximate and exact solutions of the fractional-order delay differential equations using Legendre pseudo-spectral method," International Journal of Pure and Applied Mathematics, vol. 74, no. 3, pp. 287-297, 2012.

[13] M. M. Khader, N. H. Sweilam, and A. M. S. Mahdy, "An efficient numerical method for solving the fractional difusion equation," Journal of Applied Mathematics and Bioinformatics, vol. 1, no. 2, pp. 1-12, 2011.

[14] N. H. Sweilam, M. M. Khader, and W. Y. Kota, "On the numerical solution of Hammerstein integral equations using Legendre approximation," International Journal of Applied Mathematical Research, vol. 1, no. 1, pp. 65-76, 2012.

[15] S. Yousefi and M. Razzaghi, "Legendre wavelets method for the nonlinear Volterra-Fredholm integral equations," Mathematics and Computers in Simulation, vol. 70, no. 1, pp. 1-8, 2005.

[16] J. C. Mason and D. C. Handscomb, Chebyshev Polynomials, Chapman \& Hall/CRC, Washington, DC, USA, 2003.

[17] M. Lakestani and M. Dehghan, "Numerical solution of fourthorder integro-differential equations using Chebyshev cardinal functions," International Journal of Computer Mathematics, vol. 87, no. 6, pp. 1389-1394, 2010.

[18] M. A. Snyder, Chebyshev Methods in Numerical Approximation, Prentice-Hall Inc., Englewood Cliffs, NJ, USA, 1966. 


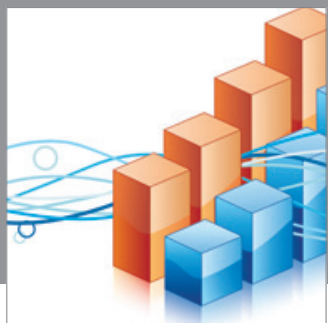

Advances in

Operations Research

mansans

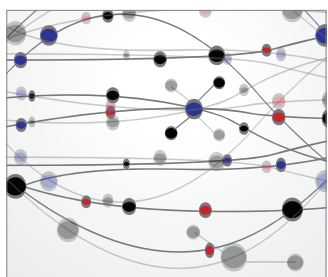

The Scientific World Journal
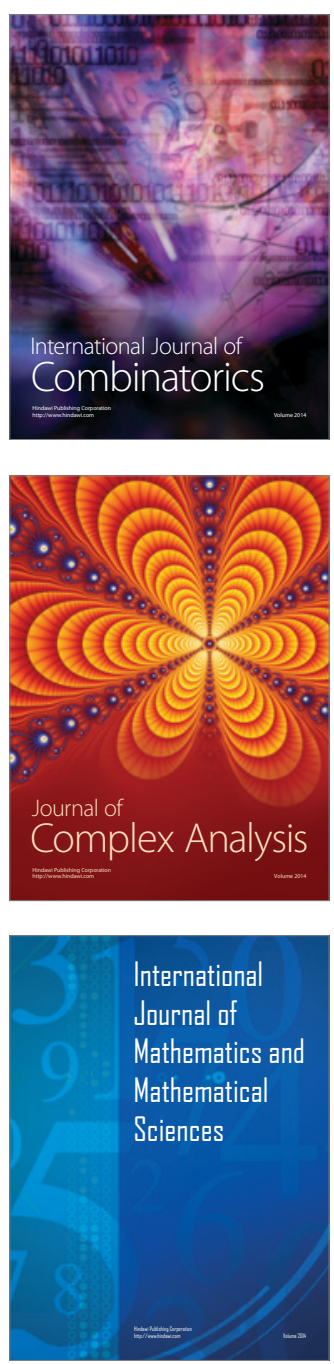
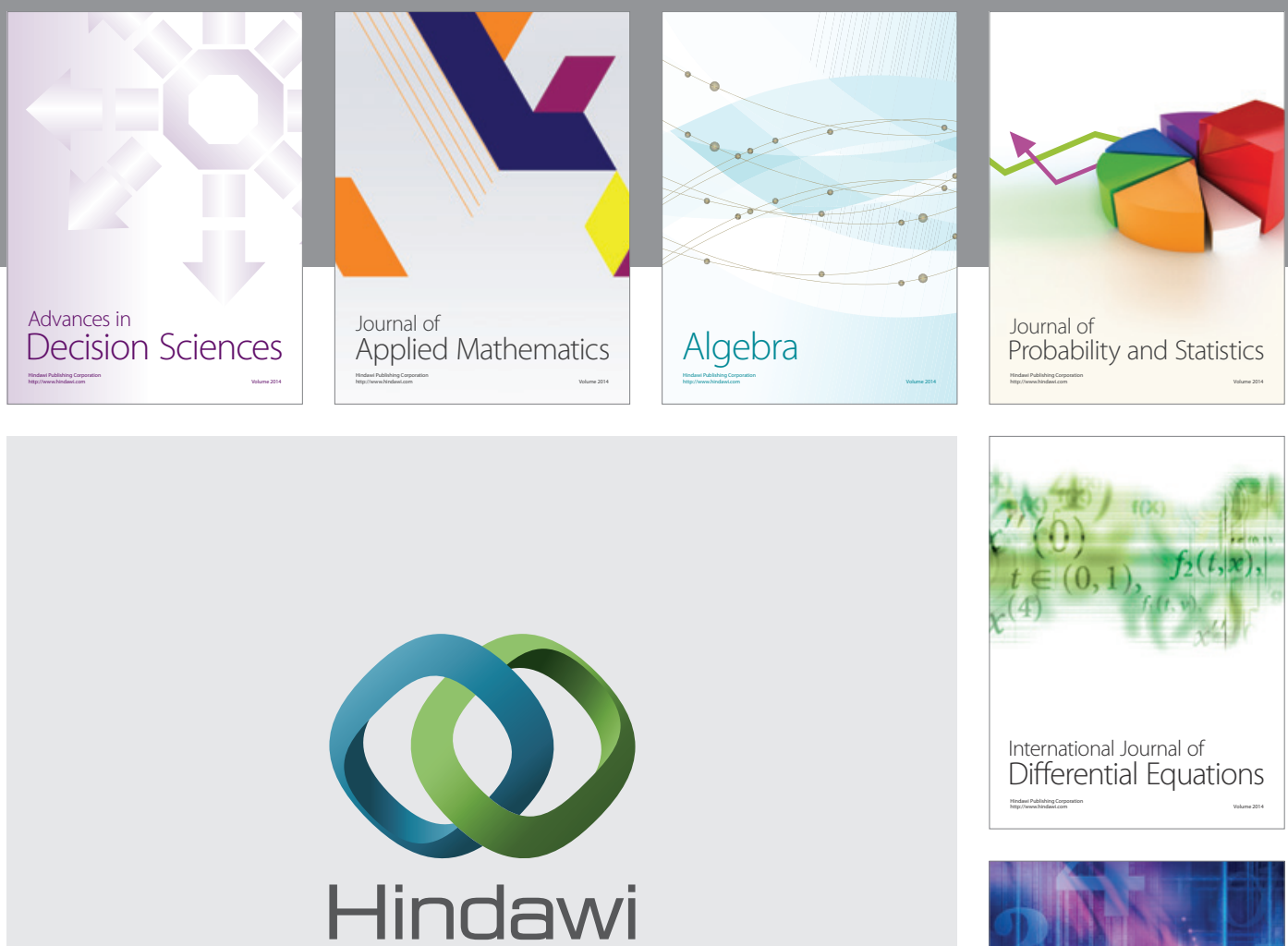

Submit your manuscripts at http://www.hindawi.com
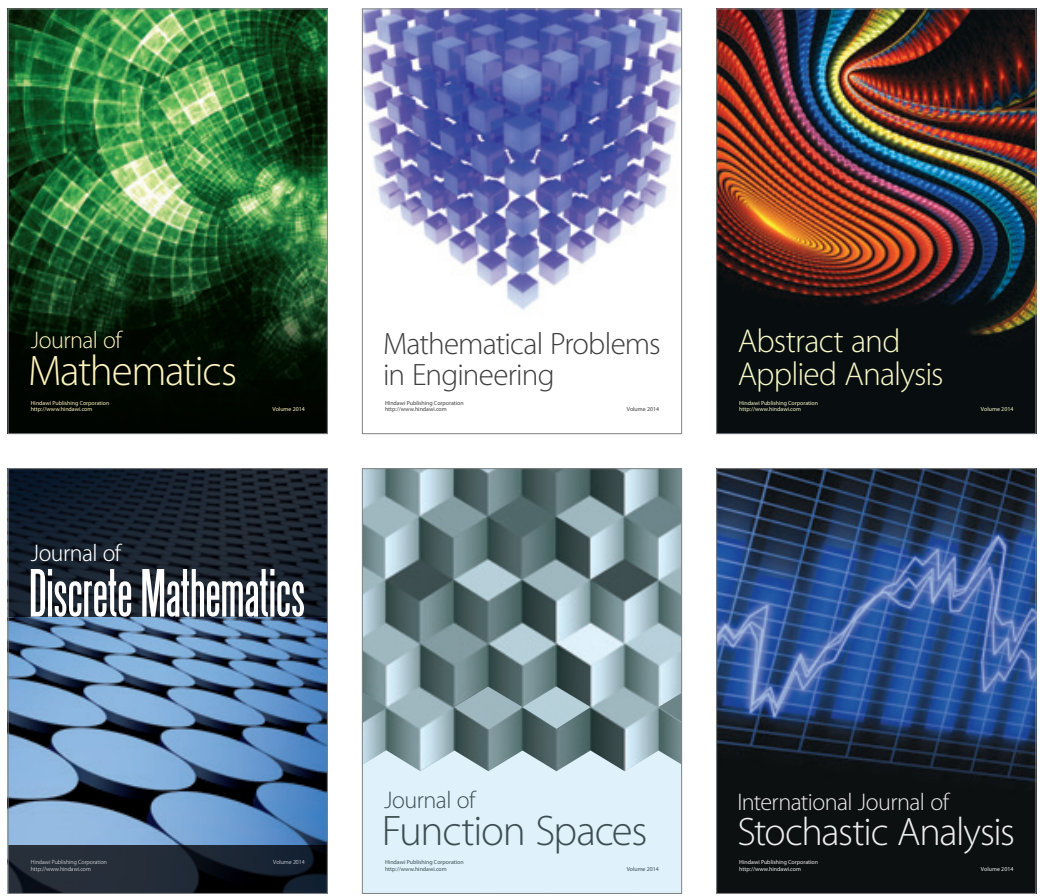

Journal of

Function Spaces

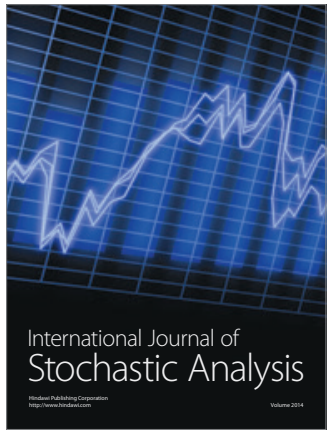

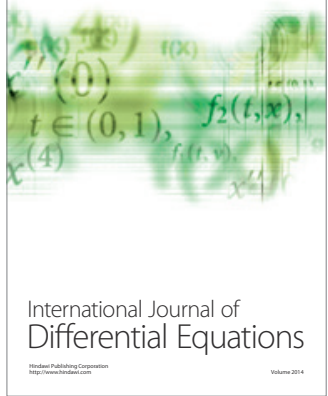
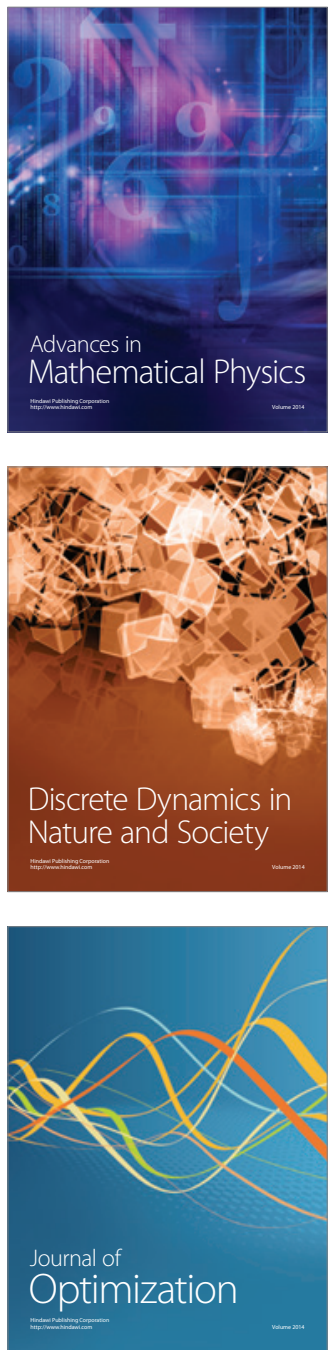